\title{
The Assessment of Serum Endocan Levels in Children With Juvenile Idiopathic Arthritis
}

\author{
Yasin YILMAZ, ${ }^{1}$ Rana BERRU DURMUŞ, ${ }^{1}$ Başak SARAÇOĞLU, ${ }^{1}$ Sezgin ŞAHIN,${ }^{2}$ Amra ADROVIC, ${ }^{2}$ \\ Kenan BARUT, ${ }^{2}$ Sevda ÖZEL YILDIZ, ${ }^{3}$ Özgür KASAPÇOPUR, ${ }^{2}$ Rukiye EKER ÖMEROĞLU ${ }^{4}$ \\ ${ }^{1}$ Department of Pediatrics, Istanbul University Istanbul Faculty of Medicine, Istanbul, Turkey \\ ${ }^{2}$ Department of Pediatrics, Division of Pediatric Rheumatology, Istanbul University Cerrahpasa Faculty of Medicine, Istanbul, Turkey \\ ${ }^{3}$ Department of Biostatistics, Istanbul University Istanbul Faculty of Medicine, Istanbul, Turkey \\ ${ }^{4}$ Department of Pediatrics, Division of Pediatric Rheumatology, Istanbul University Istanbul Faculty of Medicine, Istanbul, Turkey
}

\begin{abstract}
Objectives: This study aims to evaluate the levels of serum endocan in children with juvenile idiopathic arthritis (JIA).

Patients and methods: Sixty-seven children with JIA (30 males, 37 females; mean age $10.4 \pm 4.9$ years; range 2 to 18 years) and a sex- and agematched healthy control group of 39 children (16 males, 23 females; mean age $9.3 \pm 4.1$ years; range 1 to 17 years) were recruited. Patients with JIA were divided into two groups as the clinically active JIA group $(n=27)$ and inactive JIA group $(n=40)$.

Results: The median serum endocan level in patients with JIA was significantly higher than in the control group (633.75 $\mathrm{ng} / \mathrm{L}$ vs. $379.76 \mathrm{ng} / \mathrm{L}, \mathrm{p}<0.01)$ Comparison between patients with active JIA and inactive JIA was not significant in terms of endocan levels (618.70 $\mathrm{ng} / \mathrm{L}$ vs. $687.36 \mathrm{ng} / \mathrm{L}, \mathrm{p}=0.34)$. There was a weak negative correlation between Childhood Health Assessment Questionnaire scores of patients with JIA and serum endocan levels. Conclusion: The high level of serum endocan highlighted the endothelial damage in patients with JIA.

Keywords: Endothelial damage; juvenile idiopathic arthritis; serum endocan.
\end{abstract}

Juvenile idiopathic arthritis (JIA) is the most commonly seen rheumatic disease in childhood. The pathophysiology of JIA is based on chronic idiopathic synovitis characterized by synovial hyperplasia and inflammation. ${ }^{1}$ The presence of antinuclear antibody, rheumatoid factor, and other antibodies supports the autoimmune process in the etiopathogenesis. ${ }^{2}$

Recently, it has been mentioned that cardiovascular disease risk increased for endothelial dysfunction in patients with JIA. Increased risk of cardiovascular disease seen in the long term is associated with prominent vascular inflammation. ${ }^{3}$
Endocan is an endothelial cell-derived dermatan sulfate molecule that plays a key role in vascular inflammation. ${ }^{4,5}$ Serum levels of endocan increase in inflammatory processes such as endothelial activation and neovascularization. ${ }^{6}$ It has also been shown that serum endocan levels rise in inflammatory diseases such as inflammatory bowel disease (IBD) and Behçet's disease., ${ }^{7,8}$ Relationships between cardiovascular disease risk and serum endocan levels in patients with chronic diseases such as chronic kidney disease (CKD) and psoriasis have also been reported., ${ }^{9,10}$ Therefore, in this study, we aimed to evaluate the levels of serum endocan in children with JIA. 


\section{PATIENTS AND METHODS}

The study was a two-center, cross-sectional and observational study conducted in Istanbul Medical Faculty and Cerrahpasa Medical Faculty Pediatric Rheumatology Outpatient Clinics between August 2015 and June 2016. Children with JIA who were under follow-up at these polyclinics for at least six months were reviewed for the study. A total of 67 patients with JIA (30 males, 37 females; mean age $10.4 \pm 4.9$ years; range 2 to 18 years) were included in the study group after reviewing patients' charts, drawing online patients' records, and taking serum samples.

Institutional Review Board (Istanbul University Istanbul Medical Faculty Clinical Research Ethic Committee) approval was obtained for this study (No. 2015/1014). A written informed consent was obtained from the parent/guardian of each patient. The study was conducted in accordance with the principles of the Declaration of Helsinki.

The diagnosis of JIA was established according to International League of Associations for Rheumatology criteria. ${ }^{11}$ Exclusion criteria were presence of acute infection (i.e. upper or lower respiratory tract infection, urinary tract infection, acute gastroenteritis) or a history of any other rheumatic disorders.

After recruiting the study group, a sex- and age-matched healthy control group of 39 children (16 males, 23 females; mean age 9.3 \pm 4.1 years; range 1 to 17 years) without any acute or chronic disease was included once obtaining a written informed consent from each parent/guardian.

All patients were introduced to the research by the pediatric rheumatologist who was responsible for recruitment. Childhood Health Assessment Questionnaire (CHAQ) scores, which measure functional ability in daily living activities, were calculated when patients answered the required questions.

Patients in the study group were divided into two groups according to the American College of Rheumatology provisional criteria for defining clinical inactive disease (absence of active arthritis, duration of morning stiffness of $<15$ minutes, and inflammatory biomarkers within normal limits). ${ }^{12}$ Serum endocan levels were appropriately measured using a 2-box kit (Sunred
Biological Technology, SRB/Shanghai, China) enzyme-linked immunosorbent assay.

\section{Statistical analysis}

Data were collected retrospectively from study files, laboratory results, and serum endocan level measurements from blood samples. IBM SPSS version 21.0 (IBM Corp., Armonk, NY, USA) was used for statistical analysis. Descriptive analysis (mean, standard deviation, median, minimum, maximum, $25^{\text {th }}-75^{\text {th }}$ percentile) of quantitative variables was performed. The Kolmogorov-Smirnov test was used to assess the normality of sample distribution. For comparison of two groups, Student's t-test was used for normally distributed variables and the MannWhitney $U$ test was used for non-normally distributed variables. The Chi-square test was performed for categorical variables. To evaluate the relationship between continuous variables, Pearson's test (correlation coefficient: $r$ ) was run for normally distributed variables and Spearman's test (correlation coefficient: $r s$ ) was run for non-normally distributed variables. Results were assessed within 95\% confidence interval and $\mathrm{p}<0.05$ was considered statistically significant.

\section{RESULTS}

Demographic features of JIA patients and healthy controls were given in Table 1 . According to the American College of Rheumatology provisional criteria for defining clinical inactive disease, $40 \%(n=27)$ of patients had clinically active and $60 \%(n=40)$ had inactive JIA. The most common subtype of JIA was the oligoarticular type $(n=45 ; 67 \%)$, followed by the polyarticular type $(\mathrm{n}=14 ; 20 \%)$. The mean age at diagnosis was $7.4 \pm 4.6$ years, and the mean disease duration was $3.1 \pm 2.8$ years (Table 1 ). Twelve patients had comorbidities during the study (allergic rhinitis, osteoporosis, growth retardation, attention deficit hyperactivity disorder, cataract, gastroesophageal reflux, anemia, nephrotic syndrome, and diabetes mellitus). We also performed statistical analysis after removal of patients with comorbidities. Fifty-five percent of patients with active JIA had one joint with active arthritis, whereas the remainder had two or more joints with active inflammation. Regarding treatment, 10 patients did not take any medication, 18 patients were 


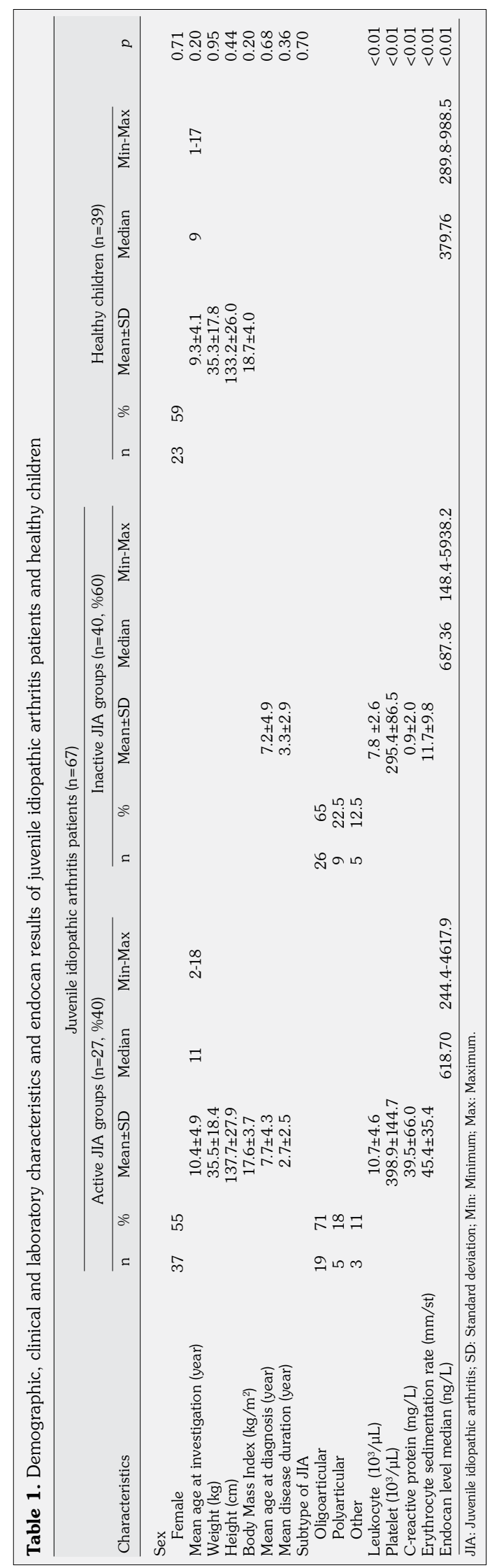

taking multi-drug therapy, and 39 patients were receiving monotherapy.

We used non-parametric tests for further analysis because the results of the analysis for the normality of serum endocan level distribution showed non-homogeneous distribution. The median value of serum endocan level in the control group was $379.76 \mathrm{ng} / \mathrm{L}$ (25 $5^{\text {th }}$ percentile $323.23 \mathrm{ng} / \mathrm{L} ; 7^{\text {th }}$ percentile $\left.469.70 \mathrm{ng} / \mathrm{L}\right)$, whereas it was $633.75 \mathrm{ng} / \mathrm{L}\left(25^{\text {th }}\right.$ percentile $398.61 \mathrm{ng} / \mathrm{L} ; 7^{\text {th }}$ percentile $\left.2181.89 \mathrm{ng} / \mathrm{L}\right)$ in the study group $(z=-4.1 ; p<0.01)$. Within the study group, the median serum endocan level in the active JIA group was $618.70 \mathrm{ng} / \mathrm{L}\left(25^{\text {th }}\right.$ percentile $398.61 \mathrm{ng} / \mathrm{L} ; 7^{\text {th }}$ percentile $\left.1457.67 \mathrm{ng} / \mathrm{L}\right)$, whereas it was $687.36 \mathrm{ng} / \mathrm{L}\left(25^{\text {th }}\right.$ percentile $405.20 \mathrm{ng} / \mathrm{L} ; 7^{\text {th }}$ percentile $3188.28 \mathrm{ng} / \mathrm{L}$ ) in the inactive JIA group $(z=-0.94 ; p=0.34)$ (Table 1, Figure 1).

Serum endocan level of patients without therapy was $471.37 \mathrm{ng} / \mathrm{L}$, whereas it was $686.42 \mathrm{ng} / \mathrm{L}$ in patients given monotherapy and $1086.15 \mathrm{ng} / \mathrm{L}$ in patients who took multiple-drug therapy $(p=0.28)$. The highest level of serum endocan in the study group was detected in patients with polyarticular JIA ( $\mathrm{n}=14$; $1788.74 \mathrm{ng} / \mathrm{L}$ ), while it was $633.75 \mathrm{ng} / \mathrm{L}$ in patients with oligoarticular JIA $(n=45)$. Because JIA is a heterogeneous disease, we also screened the oligoarticular type alone, which consisted of 19 and 26 patients with active and inactive JIA, respectively. The median serum endocan

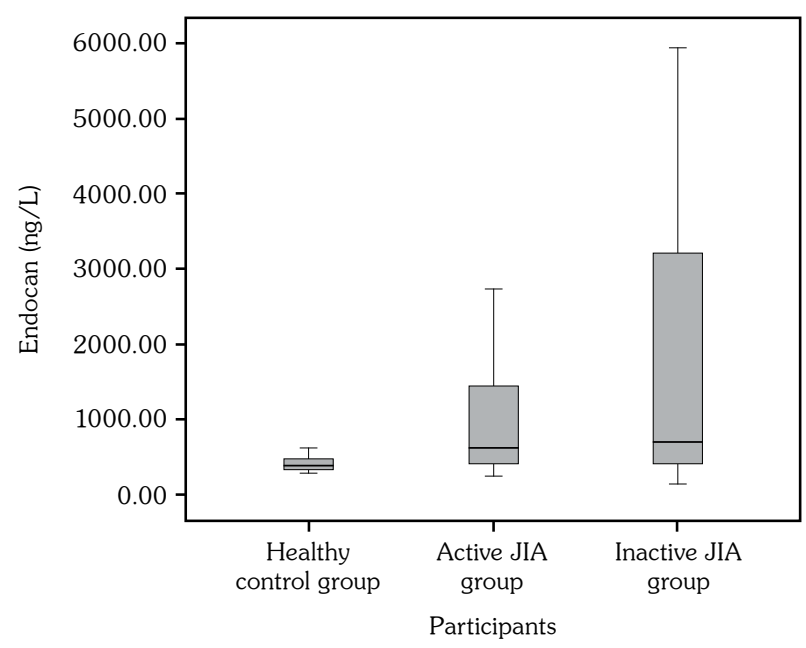

Figure 1. Serum endocan level distribution in participants. JIA: Juvenile idiopathic arthritis. 
level in the active oligoarticular JIA group was $488.90 \mathrm{ng} / \mathrm{L}$, whereas it was $687.36 \mathrm{ng} / \mathrm{L}$ in the inactive oligoarticular JIA group $(p=0.08)$.

The mean CHAQ scores of the active and inactive JIA groups were $0.63 \pm 0.45$ and $0.14 \pm 0.29$, respectively $(p<0.01)$. We investigated the correlation between serum endocan level and laboratory and clinical data within the study group. Serum endocan levels were independent from sex, age, weight, height, and Body Mass Index (BMI). There were weak negative correlations between serum endocan level and CHAQ scores and the number of joints with limitation of movement $(\mathrm{r} s=-0.25, \mathrm{p}=0.04 ; \mathrm{r} s=-0.24, \mathrm{p}=0.04$, respectively). There were no significant correlations between serum endocan level and C-reactive protein (CRP), erythrocyte sedimentation rate (ESR), or leukocyte levels in study group.

\section{DISCUSSION}

In this study, serum endocan levels of patients with JIA and a sex- and age-matched healthy control group were evaluated. We reviewed the current literature and detected no information or studies on serum endocan levels in patients with JIA. To the best of our knowledge, this is the first research in the literature to analyze serum endocan levels in patients with JIA.

Serum endocan levels were significantly higher in patients with JIA compared with the control group. However, serum endocan levels were lower in those in an active stage than in those in clinical remission. On the other hand, serum endocan levels showed a negative correlation with both limitations of movement and functional ability (CHAQ scores).

Although the level of serum endocan in patients with active JIA was low, there were no correlations with endocan levels and inflammatory biomarkers (CRP, ESR) and number of cases of active arthritis. These findings might mean that vascular inflammation and systemic inflammation may not be directly associated in JIA. The high level of serum endocan in the multiple-drug therapy group and monotherapy group rather than untreated group showed that suppressed systemic inflammation with drugs may not cover endothelial activation. In contrast to acute events (inflammatory biomarkers, active arthritis), chronic events (limitation of movement and functional capacity) were found to be more correlated with endothelial dysfunction (serum endocan).

When patients with comorbidities were excluded from analyses, serum endocan levels revealed a positive correlation with both the number of active arthritis and ESRs, and this might show that serum endocan levels are associated with disease activity to some extent. However, other systemic morbidities might be involved in endothelial activation and can change the metabolism of endocan. In addition, the average serum endocan level dropped with the exclusion of patients with comorbidities, which indicates that accompanying illnesses might affect serum endocan levels in patients with JIA.

Serum endocan level may not be associated with chronicity of the disease since it was irrelevant to the overall duration of disease. However, the negative correlation between serum endocan levels and the number of immobile joints gives a clue as to its predictive value in evaluating disability in the course of disease and its prognosis.

Previously known as endothelial cell-specific molecule, endocan is a new endothelial-derived soluble dermatan sulfate proteoglycan molecule, which was cloned from human endothelial cell deoxyribonucleic acid in $1996 .{ }^{13}$ Secretion of endocan from vascular endothelial cells is controlled by several cytokines (tumor necrosis factor, interleukin-1) and growth factors. Research about endocan showed that endocan may be a marker for vascular endothelial dysfunction. ${ }^{14}$

Patients with rheumatoid arthritis (RA) are known to carry two to five-fold higher risk for cardiovascular morbidities and mortality. ${ }^{15}$ The most important factors in the pathogenesis of cardiovascular disease in patients with RA are endothelial dysfunction and vessel wall damage. ${ }^{16}$ It has been speculated that cardiovascular complications seen in patients with JIA start with vascular damage years before symptoms present. ${ }^{17}$ Accordingly, a recent study revealed that subclinical cardiovascular changes tended to present earlier, particularly in obese individuals. ${ }^{18}$

Recent research has shown a significant association between serum endocan levels and cardiovascular diseases. Relevance of serum 
endocan to cardiovascular disease and mortality has been reported in patients with CKD. ${ }^{9}$ Serum endocan levels have also been associated with cardiovascular disease and mortality in patients with psoriasis. ${ }^{10}$

Balta et al. ${ }^{8}$ demonstrated that the mean serum endocan level in patients with Behçet's disease (inflammatory vasculitis) was $1,290 \pm 600 \mathrm{ng} / \mathrm{L}$, which was significantly higher compared with the control group, and a positive correlation between endocan levels and sedimentation rate, CRP, and disease activity. However, we found no correlation between endocan levels and sedimentation rate or CRP.

There is also evidence that serum endocan levels increase in synovial tissue of patients with RA, being stimulated particularly by adiponectin. Endocan causes angiogenesis, which is the explanatory mechanism of pannus formation. ${ }^{19}$

In a study performed on children with metabolic syndrome, ${ }^{20}$ the mean serum endocan levels were documented as $2,490 \pm 820 \mathrm{ng} / \mathrm{L}$ and a positive correlation between serum endocan levels and components of metabolic syndrome such as hypertension, insulin resistance, and fasting glucose was shown. The researchers suggested that high serum endocan levels could cause endothelial dysfunction and a tendency to atherosclerosis, which in turn could result in cardiovascular complications.

In a study of patients with systemic lupus erythematosus, ${ }^{21}$ mean serum endocan levels were reported as 2,200 $\pm 1,000 \mathrm{ng} / \mathrm{L}$ and a positive correlation between serum endocan levels and several parameters such as thickness of carotid artery wall, BMI, and sedimentation rate was shown. However, we observed no correlation between serum endocan levels and BMI. The authors attributed the high level of serum endocan and its correlation with carotid intima media thickness to subclinical atherosclerosis and therefore denoted that serum endocan levels played a critical role in susceptibility to cardiovascular diseases.

In a study on patients with systemic sclerosis, ${ }^{22}$ the mean serum endocan levels were found as $2,530 \mathrm{ng} / \mathrm{mL}$. Patients with diffuse systemic sclerosis and those who presented with digital ulcers and daily Raynaud phenomenon were shown to have higher endocan levels and a positive correlation between endocan levels and duration of disease was detected. Yet in our study, there was no correlation with duration of disease.

Lastly, in a study conducted on patients with IBD, ${ }^{7}$ there was no significant correlation between disease activity and serum endocan levels despite the fact that higher endocan levels were detected in the patient group compared with the control group. We also detected higher endocan levels in our patient group, and a weak negative correlation between functional ability (CHAQ) and serum endocan levels.

Our study has some limitations. The small sample size prevented us from making certain conclusions. The overall duration of disease being slightly short and the fact that patients were not evaluated for cardiovascular disease risk are other limitations of our study. In addition, it is difficult to draw conclusions about the possible etiology and its prognostic role in the course of the disease because the study had a crosssectional design. Therefore, we cannot precisely interpret the data obtained from this study and cannot suggest that serum endocan might be considered as a diagnostic or a follow-up parameter in JIA.

In conclusion, our study showed that serum endocan levels are higher in patients with JIA compared with controls. The results of this study may indicate that patients with JIA can be evaluated in terms of endothelial dysfunction and increased cardiovascular disease risk in a more detailed manner. Multi-centered and large prospective studies may provide more accurate and substantial data on the etiology of JIA and its cardiovascular complications.

\section{Acknowledgements}

We gratefully thank Mr. David Chapman for kindly providing language revision.

\section{Declaration of conflicting interests}

The authors declared no conflicts of interest with respect to the authorship and/or publication of this article.

\section{Funding}

This study received a grant from the Istanbul University Scientific Research Project Unit (BAP) (no.56165). 


\section{REFERENCES}

1. Kahn PJ. Juvenile idiopathic arthritis - what the clinician needs to know. Bull Hosp Jt Dis (2013). 2013;71:194-9.

2. Kasapçopur Ö, Barut K. Treatment in juvenile rheumatoid arthritis and new treatment options. Turk Pediatri Ars 2015;50:1-10.

3. Rusak M, Radzikowska U, Glowinska-Olszewska B, Dobrenko E, Piotrowska-Jastrzebska J, Dabrowska $\mathrm{M}$, et al. Endothelial progenitor cell levels in juvenile idiopathic arthritis patients: effects of antiinflammatory therapies. Pediatr Rheumatol Online J 2015;13:6.

4. Sarrazin S, Adam E, Lyon M, Depontieu F, Motte V, Landolfi $C$, et al. Endocan or endothelial cell specific molecule-1 (ESM-1): a potential novel endothelial cell marker and a new target for cancer therapy. Biochim Biophys Acta 2006;1765:25-37.

5. Béchard D, Scherpereel A, Hammad H, Gentina T, Tsicopoulos A, Aumercier M, et al. Human endothelial-cell specific molecule-1 binds directly to the integrin CD11a/CD18 (LFA-1) and blocks binding to intercellular adhesion molecule-1. J Immunol 2001;167:3099-106.

6. Sarrazin S, Lyon M, Deakin JA, Guerrini M, Lassalle $\mathrm{P}$, Delehedde $\mathrm{M}$, et al. Characterization and binding activity of the chondroitin/dermatan sulfate chain from Endocan, a soluble endothelial proteoglycan. Glycobiology 2010;20:1380-8.

7. Voiosu T, Balanescu P, Benguş A, Voiosu A, Baicuş $\mathrm{CR}$, Barbu $M$, et al. Serum endocan levels are increased in patients with inflammatory bowel disease. Clin Lab 2014;60:505-10.

8. Balta I, Balta S, Koryurek OM, Demirkol S, Mikhailidis DP, Celik T, et al. Serum endocan levels as a marker of disease activity in patients with Behçet disease. J Am Acad Dermatol 2014;70:291-6.

9. Yilmaz MI, Siriopol D, Saglam M, Kurt YG, Unal HU, Eyileten $\mathrm{T}$, et al. Plasma endocan levels associate with inflammation, vascular abnormalities, cardiovascular events, and survival in chronic kidney disease. Kidney Int 2014;86:1213-20.

10. Balta I, Balta S, Demirkol S, Mikhailidis DP, Celik $\mathrm{T}$, Akhan M, et al. Elevated serum levels of endocan in patients with psoriasis vulgaris: correlations with cardiovascular risk and activity of disease. $\mathrm{Br} J$ Dermatol 2013;169:1066-70.

11. Petty RE, Southwood TR, Manners P, Baum J, Glass $\mathrm{DN}$, Goldenberg $\mathrm{J}$, et al. International League of Associations for Rheumatology classification of juvenile idiopathic arthritis: second revision, Edmonton, 2001. J Rheumatol 2004;31:390-2.

12. Wallace CA, Giannini EH, Huang B, Itert L, Ruperto $\mathrm{N}$. American College of Rheumatology provisional criteria for defining clinical inactive disease in select categories of juvenile idiopathic arthritis. Arthritis Care Res (Hoboken) 2011;63:929-36.

13. Lassalle P, Molet S, Janin A, Heyden JV, Tavernier $\mathrm{J}$, Fiers W, et al. ESM-1 is a novel human endothelial cell-specific molecule expressed in lung and regulated by cytokines. J Biol Chem 1996;271:20458-64.

14. Balta S, Mikhailidis DP, Demirkol S, Ozturk C, Kurtoglu E, Demir M, et al. Endocan--a novel inflammatory indicator in newly diagnosed patients with hypertension: a pilot study. Angiology 2014;65:773-7.

15. Distler JH, Beyer C, Schett G, Lüscher TF, Gay S, Distler O. Endothelial progenitor cells: novel players in the pathogenesis of rheumatic diseases. Arthritis Rheum 2009;60:3168-79.

16. van Zonneveld AJ, de Boer HC, van der Veer EP, Rabelink TJ. Inflammation, vascular injury and repair in rheumatoid arthritis. Ann Rheum Dis 2010;69:57-60.

17. Rusak M, Radzikowska U, Glowinska-Olszewska B, Dobrenko E, Piotrowska-Jastrzebska J, Dabrowska $\mathrm{M}$, et al. Endothelial progenitor cell levels in juvenile idiopathic arthritis patients: effects of antiinflammatory therapies. Pediatr Rheumatol Online $\mathrm{J}$ 2015;13:6.

18. Glowinska-Olszewska B, Bossowski A, Dobrenko E, Hryniewicz A, Konstantynowicz J, Milewski R, et al. Subclinical cardiovascular system changes in obese patients with juvenile idiopathic arthritis. Mediators Inflamm 2013;2013:436702.

19. Kim KS, Lee YA, Ji HI, Song R, Kim JY, Lee SH, et al. Increased expression of endocan in arthritic synovial tissues: effects of adiponectin on the expression of endocan in fibroblast-like synoviocytes. Mol Med Rep 2015;11:2695-702.

20. Halici I, Palabiyik SS, Guducu-Tufekci F, OzbekBilgin A, Cayir A. Endothelial dysfunction biomarker, endothelial cell-specific molecule-1, and pediatric metabolic syndrome. Pediatr Int 2016;58:1124-9.

21. Icli A, Cure E, Cure MC, Uslu AU, Balta S, Mikhailidis DP, et al. Endocan Levels and Subclinical Atherosclerosis in Patients With Systemic Lupus Erythematosus. Angiology 2016;67:749-55.

22. Balanescu P, Ladaru A, Balanescu E, Voiosu T, Baicus C, Dan GA. Endocan, Novel Potential Biomarker for Systemic Sclerosis: Results of a Pilot Study. J Clin Lab Anal 2016;30:368-73. 